\title{
The Unique Mechanism of SNX9 BAR Domain for Inducing Membrane Tubulation
}

\author{
Joohyun Park ${ }^{1,2,3}$, Haiyan Zhao ${ }^{1,3}$, and Sunghoe Chang ${ }^{1,2, *}$
}

Sorting nexin 9 (SNX9) is a member of the sorting nexin family of proteins and plays a critical role in clathrinmediated endocytosis. It has a Bin-Amphiphysin-Rvs (BAR) domain which can form a crescent-shaped homodimer structure that induces deformation of the plasma membrane. While other BAR-domain containing proteins such as amphiphysin and endophilin have an amphiphatic helix in front of the BAR domain which plays a critical role in membrane penetration, SNX9 does not. Thus, whether and how SNX9 BAR domain could induce the deformation of the plasma membrane is not clear. The present study identified the internal putative amphiphatic stretch in the $1^{\text {st }} \alpha$-helix of the SNX9 BAR domain and proved that together with the $\mathrm{N}$ terminal helix $\left(\mathrm{H}_{0}\right)$ region, this internal putative amphiphatic stretch is critical for inducing membrane tubulation. Therefore, our study shows that SNX9 uses a unique mechanism to induce the tubulation of the plasma membrane which mediates proper membrane deformation during clathrinmediated endocytosis.

\section{INTRODUCTION}

Sorting nexin 9 (SNX9) initially was identified as a protein that interacts with the metalloproteases MDC9 and MDC15 (Howard et al., 1999). It is involved in clathrin-mediated endocytosis (CME), participates in actin rearrangement, and regulates various steps of vesicle transport (Howard et al., 1999; Lundmark and Carlsson, 2003; 2009; Yarar et al., 2007). SNX9 has four domains, which have different features from each other. The $\mathrm{SH} 3$ domain of SNX9 binds to proline rich domain (PRD)containing proteins, such as dynamin, N-WASP, and ACK2 (Lin et al., 2002; Shin et al., 2008; Soulet et al., 2005). The low complex (LC) region binds to the Arp $2 / 3$ complex involved in the actin nucleating process and also to clathrin and AP-2 involved

${ }^{1}$ Department of Physiology and Biomedical Sciences, ${ }^{2}$ Biomembrane Plasticity Research Center, Seoul National University College of Medicine, Seoul 110-799, Korea, ${ }^{3}$ These authors contributed equally to this work.

*Correspondence: sunghoe@snu.ac.kr

Received 12 August, 2014; revised 28 August, 2014; accepted 2 September, 2014; published online 26 September, 2014

Keywords: amphiphatic helix, BAR domain, clathrin-mediated endocytosis, invagination, tubulation in the CME pathway. The phox homology (PX) domain binds to phosphatidylinositol 4-phosphate 5 kinases [Ptdlns (4) P-5kinase], which regulates the tubulation activity of SNX9 (Shin et al., 2008; Pylypenko et al., 2007). The Bin-Amphiphysin-Rvs (BAR) domains establish a crescent-shaped homodimer, sense and generate positive membrane curvature, and induce membrane tubulation (Gallop and McMahon, 2005; Habermann, 2004; Peter et al., 2004). Recently, it was discovered that the PX and BAR domains of SNX9 are essential for its membrane modulating mechanism (Pylypenko et al., 2007; Ren et al., 2006).

BAR domain-containing proteins such as amphiphysin and endophilin have $\alpha$-helical structures that function in endocytosis, actin regulation and signaling (Dawson et al., 2006; Futterer and Machesky, 2007; Gallop et al., 2013; Itoh and De Camilli, 2006; Wang et al., 2008). These proteins contain N-terminal amphiphatic helix in front of the BAR domain, and by inserting this helix into one leaflet of the bilayer, they impress their own shape onto the membrane in order to create membrane bending (Gallop et al., 2006). Subsequently, membrane bending induces membrane tubulation, which is formed in the narrow neck region of the nascent vesicle (Gallop et al., 2006; Masuda et al., 2006).

Unlike other BAR domain containing proteins, however, the BAR domain of SNX9 is found at the C-terminus of the protein, and it does not contain an immediate upstream amphiphatic helix in front of the BAR domain (Pylypenko et al., 2007). Therefore, how the BAR domain of SNX9 mediates membrane bending is not fully understood (Pylypenko et al., 2007; Shin et al., 2007; Yarar et al., 2007).

Here, we provide evidence showing that together with the $\mathrm{N}$ terminal helix $\left(\mathrm{H}_{0}\right)$ region, the internal putative amphiphatic stretch in the $1^{\text {st }} \alpha$-helix of the SNX9 BAR domain is critical for inducing membrane tubulation. Therefore, our study shows that SNX9 uses a unique mechanism to induce membrane tubulation which may ensure proper membrane invagination during CME.

\section{MATERIALS AND METHODS}

DNA Constructs and antibody

SNX9 was amplified by PCR and the PCR product was subcloned into pEGFP vector (Clontech, USA). The mutation constructs were made by QuickChange Site-Directed Mutagenesis Kit (Stratagene, USA). All DNA constructs were verified by DNA sequencing. Primary antibody was used anti-GFP (Abcam, UK) and anti-HA (Covance, USA). Secondary antibody was 
A

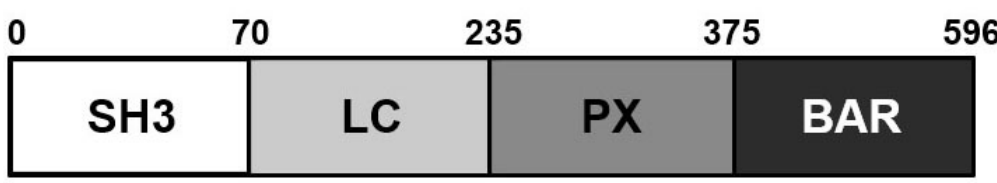

SNX 9

B

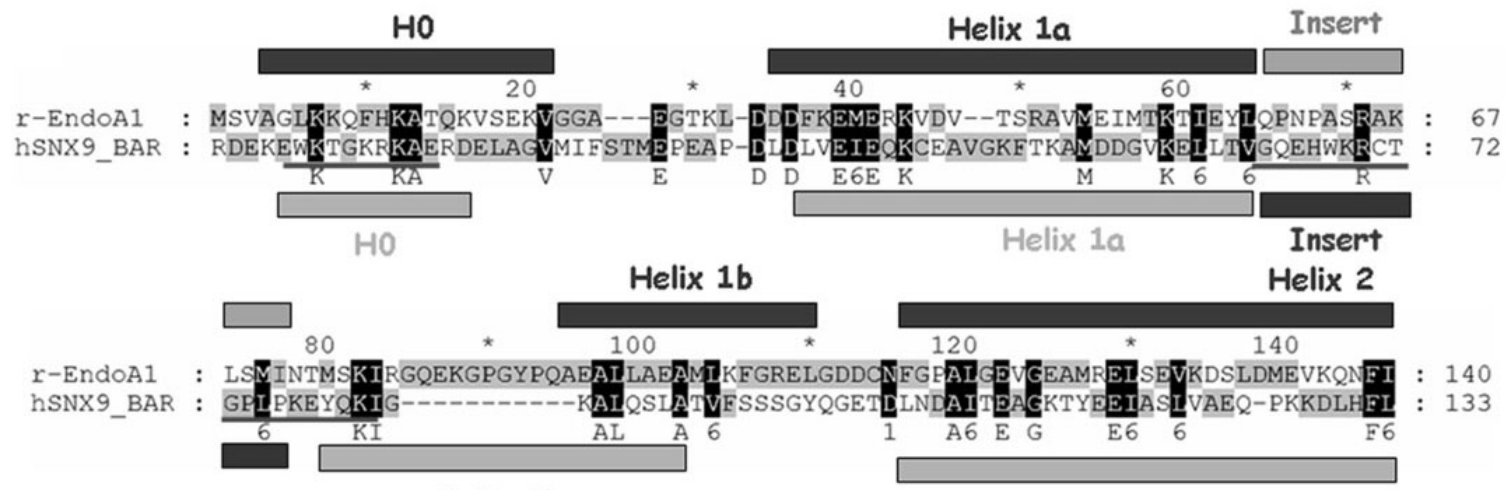

Helix 1b

Helix 2

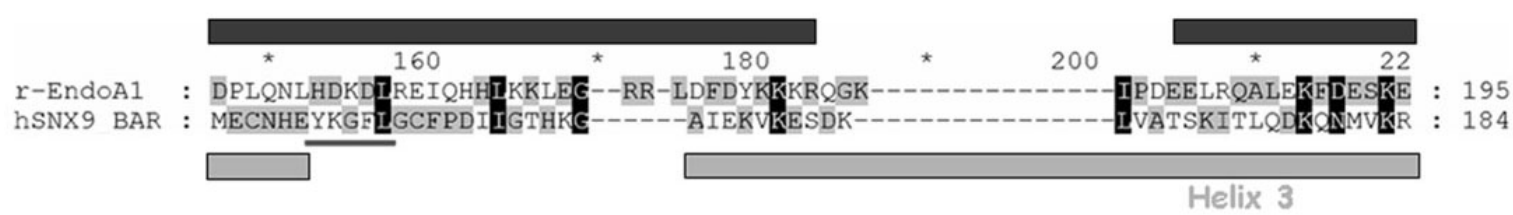

Helix 3

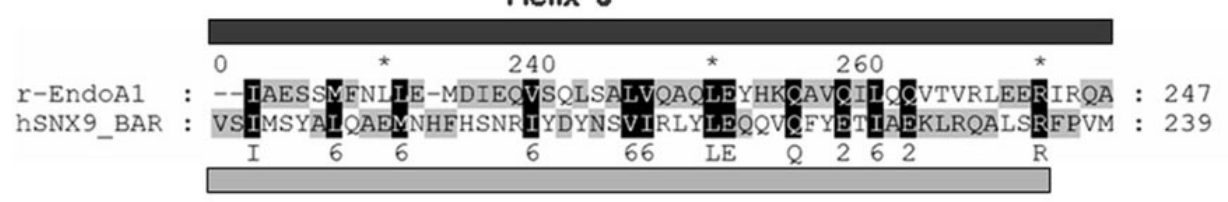

Fig. 1. SNX9 has a functional BAR domain. (A) SNX9 domain structure. Unlike N-BAR protein, endophilin, SNX9 has a C-terminal BAR domain. (B) Multiple sequence alignment of BAR domains in human SNX9 and rat endophilin A1. Conserved amino-acids residues are highlighted with black boxes. Secondary structure of them was determined from the crystal structure. An asterisk $\left(^{\star}\right)$ indicate the amino-acid residues involved in the dimerization, which is related to BAR-BAR contacts. Symbols of consensus sequence are: 1, D/N; 2, E/Q; and 6, hydrophobic.

used HRP-conjugated anti-rabbit and anti-mouse (Jackson ImmunoResearch, USA). All other reagents were from Sigma (USA).

\section{Mutagenesis}

The BAR domain mutant constructs of SNX9 were made by two complimentary oligonucleotides containing the desired mutation, flanked by unmodified nucleotide sequence. After finishing PCR reaction using SNX9 as a template, add Dpn I restriction enzyme (NEB, UK) to each PCR product to digest the parental plasmid. Subsequently, those products were transfer to the competent cell $(\mathrm{DH} 5 \alpha)$ and each products plate on agar plates containing appropriate antibiotic for plasmid vector and incubate at $37^{\circ} \mathrm{C}$ for more than $16 \mathrm{~h}$.

Cell culture and transfection

HEK293T and COS-7 cell were cultured at $37^{\circ} \mathrm{C}$ and $5 \% \mathrm{CO}_{2}$ in Dulbecco's modified Eagle's medium (Invitrogen, USA) supple- mented with $10 \%$ fetal bovine serum (Hyclone, USA). Transfection was carried out using Lipofectamine 2000 (Invitrogen), and cells were observed after 16-24 h.

Cell imaging and image analysis

For imaging, cells were fixed in 4\% formaldehyde, $4 \%$ sucrose in phosphate-buffered saline (PBS: $137 \mathrm{mM} \mathrm{NaCl}, 2.7 \mathrm{mM} \mathrm{KCl}, 10$ $\mathrm{mM} \mathrm{Na}_{2} \mathrm{HPO}_{4}, 2 \mathrm{mM} \mathrm{KH} \mathrm{PO}_{4}, \mathrm{pH} 7.4$ ) for 15 min. Immediately before image acquisition, cells were mounted on the slide glass. Fluorescence images were acquired on a Olympus IX-71 inverted microscope with 100X, 1.4 NA oil lens using a CoolSNAP-HQ CCD camera (Roper Scientific, USA) driven by MetaMorph Imaging Software (Molecular Devices, USA) with a GFP optimized filter set (Omega Optical, USA). The degree of tubulation was analyzed in MetaMorph software. Three independent experiments were performed and the data were pooled and tubulation degrees were compared with SNX9 full-length:'+++' indicates the similar degree of tubulation with SNX9 full-length, '++' indi- 
Table 1. The degree of tubule formation of various SNX9 BAR domain point mutants [X to glutamic acid (E)]

\begin{tabular}{cccc}
\hline SNX9 construct & Effect on tubules & SNX9 construct & Effect on tubules \\
\hline WT & +++ & & \\
W362E & $-/+$ & H423E & $-1+$ \\
K363E & $-/+$ & W424E & +++ \\
G365E & ++ & K425E & ++ \\
K366E & $-/+$ & R426E & ++ \\
R367E & $-/+$ & L431E & +++ \\
K368E & +++ & K433E & ++ \\
A369E & + & A437E & ++ \\
V419E & ++ & K497E & ++ \\
\hline
\end{tabular}

A

\begin{tabular}{|c|c|c|c|c|c|c|}
\hline Helixo & Helix1a & Insert & Helix1b & Helix2 & & Helix3 \\
\hline 357 & \multicolumn{4}{|c|}{ 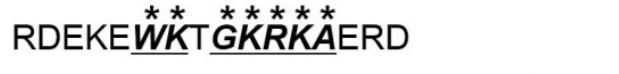 } & \multicolumn{2}{|l|}{372} \\
\hline 416 & \multicolumn{4}{|c|}{ 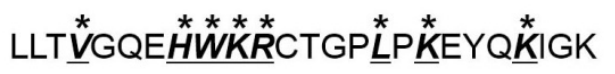 } & \multicolumn{2}{|c|}{440} \\
\hline 490 & \multicolumn{4}{|c|}{ ECNHEY ${ }^{*}$ GFLGCFP } & 504 & \\
\hline
\end{tabular}

B

SNX9 G365E K366E H423E W424E K425E K433E K497E W362E

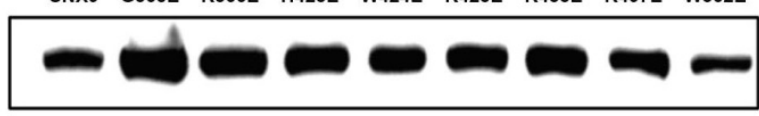

SNX9 K363E R367E R368E A369E V419E R426E L431E K437E
Fig. 2. The candidate sites of SNX9 BAR domain point mutation. The target sites of BAR domain point mutation. (A) SNX9 BAR domain has three major functional components: the short $\mathrm{N}$-terminal helix region $\left(\mathrm{H}_{0}\right)$, the BAR domain and an insert putative amphiphatic helix in the middle of helix1. We selected candidate sites from both of $\mathrm{H}_{0}$ and insert region, and then point mutated to Glutamic acid, E. (B) Protein expressions of point mutants tagged with pEGFP. HEK 293T cells were transfected with various mutants and immunoblotted using polyclonal anti-GFP antibody. All point mutants were expressed well on their correct sizes. cate more than $50 \%$ of tubular structure remains, which compare to SNX9 full-length, ' + ' indicates $30-50 \%$ of tubular structure remains, compare to SNX9 full-length, '-/+', indicate rarely found tubular structure.

\section{RESULTS}

SNX9 has a functional BAR domain

SNX9 is composed of four functional domains: N-terminal SH3, LC, PX, and C-terminal BAR domains (Fig. 1A). While other BAR domain-containing proteins such as amphiphysin and endophilin have the BAR domain in their N-terminus, the SNX9 BAR domain is located in its C-terminus. Figure $1 B$ shows a multiple alignment of the human SNX9 BAR domain and rat endophilin A1 BAR domain. We found that in addition to the $\mathrm{N}$ terminal short helix region $\left(\mathrm{H}_{0}\right)$, SNX9 has additional insert region in the middle of helix1. This extra insert region is located in the middle of its membrane binding surface and contains hydrophobic, acid and basic amino acids, which make it a putative amphiphatic region.

We set out to identify the amino acid residue(s) of this insert region which is (are) critical for this membrane tubulation activity by expressing various point mutants. We selected candidate sites in both the $\mathrm{N}$-terminal helix $\left(\mathrm{H}_{0}\right)$ and the additional insert region (Fig. 2).
Membrane tubulating capacity by SNX9 full-length and BAR domain point mutation constructs

First, we changed candidate amino acids in the $\mathrm{N}$-terminal helix $\left(\mathrm{H}_{0}\right)$ and the insert region to negatively charged glutamic acid. Because the plasma membrane has a negative charge, this mutation was expected not to bind to the target amino acid in the plasma membrane. Consistent with previous reports, SNX9 fulllength tagged with $\mathrm{pEGFP}$ formed long membrane tubules (Fig. $3 A)$. When we transfected with various point mutants, we found that the point mutants W362E, K363E, K366E, and R367E in the $\mathrm{N}$-terminal helix $\left(\mathrm{H}_{0}\right)$ site and $\mathrm{H} 423 \mathrm{E}$ in the additional insert region did not make tubular structures while the other point mutants induced tubulation well, although the degree of tubulation was different from each other. These results suggest that both the N-terminal helix $\left(\mathrm{H}_{0}\right)$ and the additional insert region are involved in the tubulation event. Table 1 summarizes the results of the point mutation experiments.

N-terminal helix $\left(\mathrm{H}_{0}\right)$ is critical for inducing membrane tubulation

Based on the above results, we selected five candidate sites for another point mutation. Because glutamic acid has a negative charge, the effect could be exaggerated. Therefore, we changed the five selected target sites to alanine (A) or glutamine (Q), which has a neutral charge. The results are shown in Fig. 4. Only the N-terminal helix $\left(\mathrm{H}_{0}\right)$ mutants W362A, W362Q, K363A, 
SNX9 BAR Domain on Membrane Tubulation

Joohyun Park et al.

Table 2. The degree of tubule formation of various SNX9 BAR domain point mutants [X to glutamine $(Q)$ or Alanine $(A)]$

\begin{tabular}{cccc}
\hline SNX9 construct & Effect on tubules & SNX9 construct & \\
WT & +++ & Effect on tubules & W362A \\
W362Q & + & K363A \\
K363Q & + & $K 366 A$ & $-/+$ \\
K366Q & + & R367A \\
R367Q & ++ & H423A \\
H423Q & +++ & $-/+$ \\
+
\end{tabular}

A



B


R367E : -/+

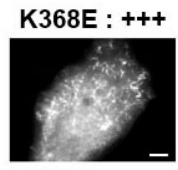

C
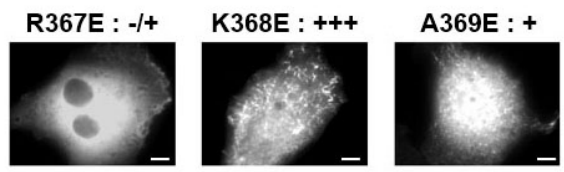

H423E : -/+
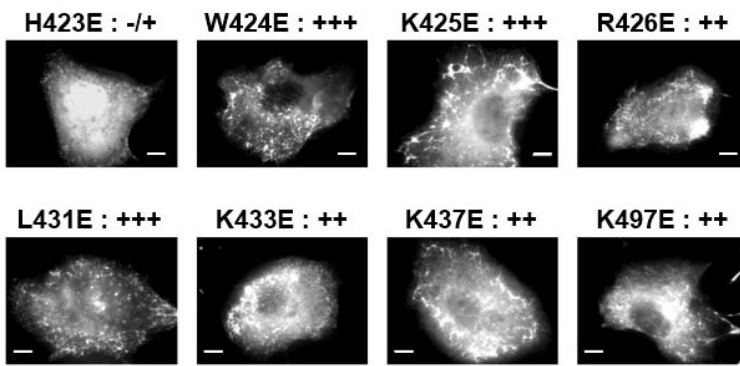

K497E : ++

Fig. 3. Membrane tubulation effect by SNX9 full-length and BAR domain point mutants. Epifluorescence micrograph of COS-7 cells transfected with GFP-SNX9 full-length $(A)$ and mutant forms $(B, C)$. (A) GFP-SNX9 created distinct elongated tubular structures. Scale bars: $10 \mu \mathrm{m}, 2 \mu \mathrm{m}$ for enlarged figures. (B, C) showed the deficient tubular structure of BAR domain point mutation constructs. The degree of tubulation indicated with following ' + ' sign: ' +++ ', similar degree of tubulation with SNX9 full-length; '++', more than $50 \%$ of tubular structure remains compare to full-length; ' + ', $30-50 \%$ of tubular structure remains compare to full-length; '- $-1+$ ', rarely found tubular structure. Note that the mutants in both of $\mathrm{H}_{0}$ and insert region showed the diffuse cytoplasmic expression pattern. See Table 1 for quantification. Scale bars are $10 \mu \mathrm{m}$.
Table 3. The degree of tubule formation in the additional insert region of SNX9 BAR domain [double-point mutation X to Alanine $(A) s$ ]

\begin{tabular}{cc}
\hline SNX9 construct & Effect on tubules \\
\hline WT & +++ \\
W424A/H423A & +++ \\
K425A/H423A & $-/+$ \\
$R 426 A / H 423 A$ & $-/+$ \\
L431A/H423A & ++ \\
K433A/H423A & ++ \\
\hline
\end{tabular}

K363Q, K366A, K366Q, and R367A failed to induce membrane tubulation. Point mutants in the additional insert region induced a tubular structure (Fig. 4 and Table 2), suggesting that the $\mathrm{N}$ terminal helix $\left(\mathrm{H}_{0}\right)$ seems to be much critical for inducing membrane tubulation.

\section{Insert region also has an effect on tubulation}

Because the H423E mutant did not induce a tubular structure while the H423A and H423Q mutants did induce a tubule well, we wondered whether the additional insert region has any roles in membrane tubulation. The following candidate sites were selected in the insert region, H423, W424, K425, R426, L431, and $\mathrm{K} 433$, and using the $\mathrm{H} 423 \mathrm{~A}$ mutant as a template, we induced a double point mutation to alanine $(A)$.

Although the single point mutants W424E, K425E, R426E, L431E, and K433E- formed membrane tubules (Fig. 3), Figure 5 shows that two of the double mutants, H423A/K425A and H423A/R426A, failed to induce a membrane tubular structure. These results show that $\mathrm{H} 423, \mathrm{~K} 425$, and $\mathrm{R} 426$ is important in inducing membrane tubular structures (Fig. 5 and Table 3).

\section{DISCUSSION}

In this study, we demonstrated that both the N-terminal helix $\left(\mathrm{H}_{0}\right)$ and the additional insert region of the SNX9 BAR domain are critical for inducing membrane tubular structures.

The SNX9 BAR domain forms a crescent-shape homodimer structure (Childress et al., 2006) which is necessary for inducing membrane tubulation because this structure forms sufficient rigidity to overcome the bending resistance of the membrane (Masuda et al., 2006; McMahon and Gallop, 2005; Zimmerberg and Kozlov, 2006). The SNX9 BAR domain also has an Nterminal helix $\left(\mathrm{H}_{0}\right)$ for membrane binding, which enhances curvature generation (Fernandes et al., 2008; Low et al., 2008). We found here that the SNX9 BAR domain has a unique appendage which is an internal putative amphiphatic stretch in the $1^{\text {st }} \alpha$-helix of the SNX9 BAR domain.

Although SNX9 does not contain an amphiphatic helix in front of the BAR domain that plays a critical role in membrane penetration, we expected that the additional insert region of the SNX9 

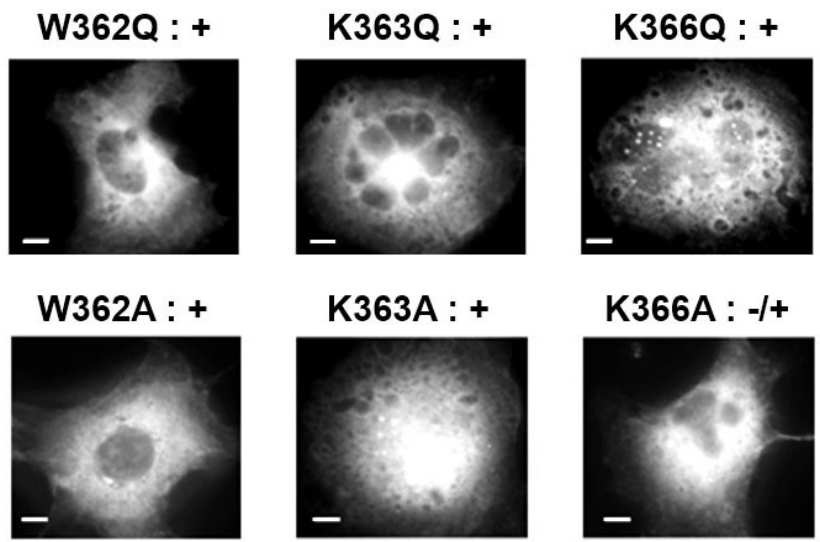

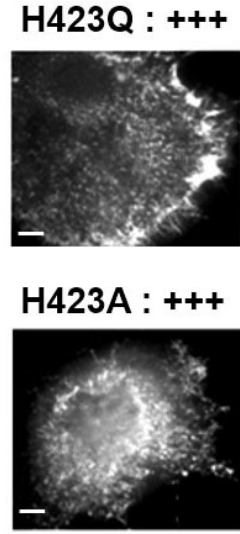

Fig. 4. N-terminal helix $\left(\mathrm{H}_{0}\right)$ is critical for inducing membrane tubulation. Epifluorescence micrographs of COS-7 cells transfected with various BAR domain point mutants. Based on the results in Fig. 3, we point mutated candidate sites to alanine (A) or glutamine (Q). See Table 2 for quantification. Scale bars, $10 \mu \mathrm{m}$.

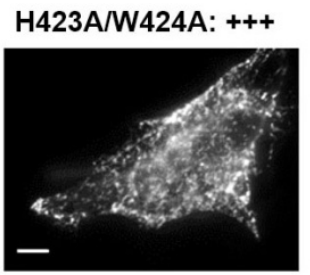

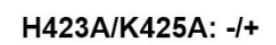



H423A/R426A: -1+

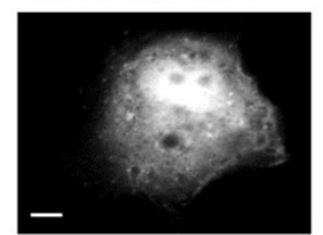

Fig. 5. Insert region also has an effect on inducing tubular structure. Epifluorescence micrograph of COS-7 cells transfected with double point mutation constructs of SNX9 BAR domains. Based on the result with an insert region point mutation construct, H423A, the following candidate sites were selected in the insert region, $\mathrm{H} 423, \mathrm{~W} 424, \mathrm{~K} 425$, R426, L431, and K433, and using the H423A mutant as a template, we induced a double point mutation to alanine $(A)$. While each single point mutant forms clearly showed the tubular structure, some of double mutant forms displayed the deficient tubule. See Table 3 for quantification. Scale bars, $10 \mu \mathrm{m}$.
BAR domain does have a critical role as a putative amphiphatic helix. Based on the results, we propose a membrane bending mechanism by SNX9. First, the N-terminally located helix $\left(\mathrm{H}_{0}\right)$ of the crescent-shape BAR homodimer senses and induces the curved plasma membrane. After that, the additional insert region penetrates the concave membrane. Then, both of them induce membrane deformation.

According to our results, even though the N-terminal helix $\left(\mathrm{H}_{0}\right)$ has more important roles in invagination, synergistic function is needed from the additional insert region for membrane invagination. Pylypenko et al. suggested that amino acid residues of the additional insert region are involved in the dimerization and are related to the hydrogen bonding of the BAR-BAR contact and the hydrophobic BAR-BAR contact (Pylypenko et al., 2007). In addition, oligomerization of Pacsin/Syndapin, an F-BAR domain containing protein family, influences tubular stabilization (Wang et al., 2009). We thus hypothesize that the additional insert region plays a critical role in membrane invagination together with the N-terminal helix $\left(\mathrm{H}_{0}\right)$ by regulating the BAR-BAR dimerization. This raises the possibility that the additional insert region is required for forming the rigid crescent-shape BAR homodimer structure rather than required for penetrating the plasma membrane. In either case, the additional insert region plays a critical role in inducing membrane tubulation although the exact working mechanism needs further studies.

\section{ACKNOWLEDGMENTS}

This work was supported by the grant funded by the Seoul National University Hospital (03-2011-0190).

\section{REFERENCES}

Childress, C., Lin, Q., and Yang, W. (2006). Dimerization is required for SH3PX1 tyrosine phosphorylation in response to epidermal growth factor signalling and interaction with ACK2. Biochem. J. 394, 693-698.

Dawson, J.C., Legg, J.A., and Machesky, L.M. (2006). Bar domain proteins: a role in tubulation, scission and actin assembly in clathrin-mediated endocytosis. Trends Cell Biol. 16, 493-498.

Fernandes, F., Loura, L.M., Chichon, F.J., Carrascosa, J.L., Fedorov, A., and Prieto, M. (2008). Role of helix 0 of the N-BAR domain in membrane curvature generation. Biophys. J. 94, 3065-3073.

Futterer, K., and Machesky, L.M. (2007). "Wunder" F-BAR domains: going from pits to vesicles. Cell 129, 655-657.

Gallop, J.L., and McMahon, H.T. (2005). BAR domains and membrane curvature: bringing your curves to the BAR. Biochem. Soc. Symp. 2005, 223-231.

Gallop, J.L., Jao, C.C., Kent, H.M., Butler, P.J., Evans, P.R., Langen, R., and McMahon, H.T. (2006). Mechanism of endophilin N-BAR domain-mediated membrane curvature. EMBO J. 25, 2898-2910.

Gallop, J.L., Walrant, A., Cantley, L.C., and Kirschner, M.W. (2013). 
Phosphoinositides and membrane curvature switch the mode of actin polymerization via selective recruitment of toca-1 and Snx9. Proc. Natl. Acad. Sci. USA 110, 7193-7198.

Habermann, B. (2004). The BAR-domain family of proteins: a case of bending and binding? EMBO Rep. 5, 250-255

Howard, L., Nelson, K.K., Maciewicz, R.A., and Blobel, C.P. (1999). Interaction of the metalloprotease disintegrins MDC9 and MDC15 with two $\mathrm{SH} 3$ domain-containing proteins, endophilin I and SH3PX1. J. Biol. Chem. 274, 31693-31699.

Itoh, T. and De Camilli, P. (2006). BAR, F-BAR (EFC) and ENTH/ANTH domains in the regulation of membrane-cytosol interfaces and membrane curvature. Biochim. Biophys. Acta $1761,897-912$

Lin, Q., Lo, C.G., Cerione, R.A., and Yang, W. (2002). The Cdc42 target ACK2 interacts with sorting nexin 9 (SH3PX1) to regulate epidermal growth factor receptor degradation. J. Biol. Chem. 277, 10134-10138.

Low, C., Weininger, U., Lee, H., Schweimer, K., Neundorf, I., BeckSickinger, A.G., Pastor, R.W., and Balbach, J. (2008). Structure and dynamics of helix-0 of the N-BAR domain in lipid micelles and bilayers. Biophys. J. 95, 4315-4323.

Lundmark, R., and Carlsson, S.R. (2003). Sorting nexin 9 participates in clathrin-mediated endocytosis through interactions with the core components. J. Biol. Chem. 278, 46772-46781.

Lundmark, R., and Carlsson, S.R. (2009). SNX9 - a prelude to vesicle release. J. Cell Sci. 122, 5-11.

Masuda, M., Takeda, S., Sone, M., Ohki, T., Mori, H., Kamioka, Y., and Mochizuki, N. (2006). Endophilin BAR domain drives membrane curvature by two newly identified structure-based mechanisms. EMBO J. 25, 2889-2897.

McMahon, H.T., and Gallop, J.L. (2005). Membrane curvature and mechanisms of dynamic cell membrane remodelling. Nature 438, 590-596.

Peter, B.J., Kent, H.M., Mills, I.G., Vallis, Y., Butler, P.J., Evans, P.R., and McMahon, H.T. (2004). BAR domains as sensors of membrane curvature: the amphiphysin BAR structure. Science 303, 495-499.

Pylypenko, O., Lundmark, R., Rasmuson, E., Carlsson, S.R., and Rak, A. (2007). The PX-BAR membrane-remodeling unit of sorting nexin 9. EMBO J. 26, 4788-4800.

Ren, G., Vaijhala, P., Lee, J.S., Winsor, B., and Munn, A.L. (2006) The BAR domain proteins: molding membranes in fission, fusion, and phagy. Microbiol. Mol. Biol. Rev. 70, 37-120.

Shin, N., Lee, S., Ahn, N., Kim, S.A., Ahn, S.G., YongPark, Z., and Chang, S. (2007). Sorting nexin 9 interacts with dynamin 1 and N-WASP and coordinates synaptic vesicle endocytosis. J. Biol. Chem. 282, 28939-28950.

Shin, N., Ahn, N., Chang-lleto, B., Park, J., Takei, K., Ahn, S.G., Kim, S.A., Di Paolo, G., and Chang, S. (2008). SNX9 regulates tubular invagination of the plasma membrane through interaction with actin cytoskeleton and dynamin 2 . J. Cell Sci. 121, 1252-1263.

Soulet, F., Yarar, D., Leonard, M., and Schmid, S.L. (2005). SNX9 regulates dynamin assembly and is required for efficient clathrinmediated endocytosis. Mol. Biol. Cell 16, 2058-2067.

Wang, Q., Kaan, H.Y., Hooda, R.N., Goh, S.L., and Sondermann, H. (2008). Structure and plasticity of endophilin and sorting nexin 9. Structure 16, 1574-1587.

Wang, Q., Navarro, M.V., Peng, G., Molinelli, E., Goh, S.L., Judson, B.L., Rajashankar, K.R., and Sondermann, H. (2009). Molecular mechanism of membrane constriction and tubulation mediated by the F-BAR protein Pacsin/Syndapin. Proc. Natl. Acad. Sci. USA 106, 12700-12705.

Yarar, D., Waterman-Storer, C.M., and Schmid, S.L. (2007). SNX9 couples actin assembly to phosphoinositide signals and is required for membrane remodeling during endocytosis. Dev. Cell $13,43-56$.

Zimmerberg, J., and Kozlov, M.M. (2006). How proteins produce cellular membrane curvature. Nat. Rev. Mol. Cell Biol. 7, 9-19. 